A N N A L E S Annales de Bretagne et des Pays de l'Ouest

\title{
Louis XIII, un roi de guerre à la conquête du pouvoir,
} 1620-1622

\section{Thierry Heckmann}

\section{Q OpenEdition \\ 1 Journals}

Édition électronique

URL : https://journals.openedition.org/abpo/4891

DOI : $10.4000 / a b p o .4891$

ISBN : 978-2-7535-8021-3

ISSN : $2108-6443$

Éditeur

Presses universitaires de Rennes

Édition imprimée

Date de publication : 20 décembre 2019

Pagination : 232-235

ISBN : 978-2-7535-8019-0

ISSN : 0399-0826

Référence électronique

Thierry Heckmann, «Louis XIII, un roi de guerre à la conquête du pouvoir, 1620-1622 », Annales de

Bretagne et des Pays de l'Ouest [En ligne], 126-4 | 2019, mis en ligne le 22 janvier 2020, consulté le 03 janvier 2023. URL : http://journals.openedition.org/abpo/4891 ; DOI : https://doi.org/10.4000/abpo. 4891 
laient ces circuits. On suit aussi avec Jacques Péret, l'itinéraire de Joseph Micheau, un capitaine rochelais qui a su tirer parti des diverses opportunités fournies par l'océan : cabotage, course, grand commerce et guerre. Menée essentiellement sur terre, la guerre investit, lors du long conflit avec l'Angleterre, la Manche et ensuite l'ensemble des océans constate Stéphane Curveillier, les deux batailles d'Arneminden et de l'Écluse marquant le début d'une politique étatique assurant une suprématie à la couronne anglaise et introduisant des stratégies nouvelles (défense des détroits, siège des villes, course, surveillance ou attaque des filets de pêche)...

Le long de ce littoral, un territoire s'individualise : les marais salants, objet de quatre contributions émanant de Jean-Claude Hocquet, Julien Briand, Alain Gallicé, Thierry Sauzeau. Il alimente un grand commerce qui le mène de la Bretagne à la Baltique avec des opérations de raffinage en Zélande pour qu'il prenne sa couleur immaculée, avant d'être vendu dans les cités du nord de l'Europe. Son importance pour la conservation des aliments et les revenus qu'il engendre en font un produit stratégique dont le commerce pendant les conflits peut être suspendu. La guerre affecte fortement ces espaces comme le montre l'enquête "Bouthillier " menée après le traité d'Utrecht pour mesurer l'état des marais salants et qui constate à la fois l'abandon d'une large partie des salines de Brouage et l'expansion de celles d'Oléron, une situation imputable nous dit Thierry Sauzeau, au retrait de l'océan mais aussi aux modalités de détention de ces marais et à la plus forte implication des îliens. Cette production réunit plusieurs catégories d'individus : les sauniers, des ouvriers spécialisés, mais pauvres et des bourgeois qui investissent dans cette production comme Robert Blanchet, membre du conseil des bourgeois de Nantes et Michel le Pensec, receveur ordinaire du duc.

Comme tous les mélanges, cette publication présente des atouts et des faiblesses : la limite de certains propos liés à la taille (imposée) des articles qui ne permet pas de développer de manière plus exhaustive, une certaine dispersion des thématiques traitées qui s'éloignent parfois des rivages atlantiques, mais une réunion d'articles qui présente aussi le grand intérêt de présenter en un seul volume de 350 pages, toutes les facettes de ces sociétés atlantiques du Moyen Âge à l'époque moderne reprises dans la conclusion qui aurait été renforcée toutefois par l'établissement en parallèle de courtes synthèses après chacune des thématiques exposées, une histoire qui, comme l'océan, n'est jamais immobile ni au repos!

Cédric JEANNEAU

AvRILLAS, Patrick, Louis XIII, un roi de guerre à la conquête du pouvoir, 1620-1622, La Geste, 2019, 321 p., nombreuses illustrations.

"Enfin, aux chastimentz il se laisse forcer. " Cette terrible constatation concerne Louis XIII et sert de légende à une gravure intitulée "Punition des villes rebelles ", que nous fait découvrir Patrick Avrillas. Son Louis XIII, roi de guerre est consacré en effet au retentissement que les arts et la littérature accordèrent aux événements militaires de 1620-1622, qui avaient eu des relents de guerre civile. Il nous 
fait découvrir en particulier l'œuvre du graveur Jean Valdor qui leur était consacrée sous le titre des Triomphes de Louis le Juste. Corneille, à qui avait été confié le commentaire de ces gravures, précisait à propos des villes rebelles : «Une juste rigueur doibt régner à son tour, et qui veut affermir un trosne légitime, doibt semer la terreur aussi bien que l'amour. " Valdor et Corneille s'exprimaient en fait un quart de siècle plus tard, en 1649. La Fronde remettait alors en cause l'autorité royale, ou du moins celle de la mère du roi Louis XIV encore tout enfant. Elle semblait un retour à la désastreuse situation que Louis XIII avait pourtant brillamment maîtrisée.

Dans un livre précédent, Louis XIII et la bataille de l'isle de Rié : 1622, les armes victorieuses de la monarchie absolue, Patrick Avrillas nous a déjà fait connaître la bataille de Rié, ou du moins son rôle-clef dans le règne de Louis XIII. L'importance donnée par la suite au siège de La Rochelle, et surtout la propagande dont l'entoura le cardinal de Richelieu, amoindrit le souvenir des premières campagnes du roi et en particulier celui de l'île de Rié (Saint-Hilaire-de-Riez, Notre-Dame et Croix-de-Vie, dép. actuel de la Vendéé), confondue même souvent avec l'île de Ré. Pourtant, avant même d'avoir donné au cardinal la place qu'il devait occuper dans la construction de la monarchie absolue, Louis XIII avait déjà su faire face aux menaces d'éclatement du royaume : de grands seigneurs défendaient alors en effet, les armes à la main, leur désir d'émancipation politique, tout comme un fort parti protestant. Et parmi tant de batailles, a clairement établi Patrick Avrillas, c'est à Rié que le roi s'est personnellement distingué en prenant un réel risque physique. Le rappel de cette témérité exceptionnelle pour un souverain régnant fonda sa gloire militaire.

En élargissant son propos au contexte militaire dans lequel s'insère cette bataille de Rié, Patrick Avrillas revient moins sur l'histoire de cette période que sur l'écho qu'on a cherché à lui donner dans l'instant même et par la suite. La monarchie en effet ne luttait pas que par les armes mais aussi par la propagande, et celle-ci s'appuyait sur tous les moyens disponibles, tout particulièrement les arts, majeurs comme mineurs. Patrick Avrillas, par l'étendue de son recensement qui témoigne de leur extraordinaire mobilisation, surprendra ici le lecteur. La gravure fut mise à contribution, mais aussi la peinture, la sculpture, les décors, l'affiche, la cartographie, le vitrail, les médailles, la littérature sous diverses formes (libelles, satires, panégyriques, tragédies, ouvrages historiques, etc.). Pour s'exprimer à son tour, c'est la tapisserie que retint Rubens lui-même, et s'il n'est pas question de musique dans ce livre, c'est peut-être que les témoignages en ont disparu, car on ne voit pas pourquoi on n'en aurait pas composé pour la gloire et l'honneur du roi. Toutes ces œuvres recourent abondamment à l'allégorie mais aussi au parallélisme des situations historiques, ainsi en est-il du Cycle de Constantin, cette tenture de huit pièces que Rubens imagina en veillant à l'actualité la plus immédiate de l'histoire de Louis XIII. Les allusions historiques, claires pour les contemporains, ont cependant fini par nous échapper. Toutefois les emprunts que les artistes se faisaient les uns aux autres ont permis à Patrick Avrillas des recoupements confirmant l'identification aux événements de 1620-1622. Mieux, les arguments qu'il donne pour identifier cette tenture comme étant celle de la chambre du roi, à Versailles, telle que la signale l'inventaire du château dressé en 1630 , convainquent les spécialistes les uns après les autres. 
Cet ouvrage permet aussi d'appréhender la question de la violence et de ses ambiguïtés. Corneille, on l'a vu, la justifiait au nom de la raison d'État, au bas d'une des gravures que Jean Valdor consacrait à la "punition des villes rebelles ", sans oser pour autant citer explicitement le cas de Nègrepelisse, qu'il avait évidemment en tête. C'est que Louis XIII, après avoir pris cette ville, en avait bel et bien ordonné la mise à feu et à sang. Qu'on se rappelle les horreurs de la guerre décrites par les gravures du lorrain Jacques Callot. C'était sur un autre théâtre, certes, mais dans ce même début du XVII ${ }^{e}$ siècle. Du pillage, l'on glissait facilement à la violence sur les vaincus. Le roi devait pourtant la réguler dans les justes limites du droit dont il était le garant. Louis XIII gagna du reste en ces années 1620-1622 le titre de Louis le Juste. Il ne pouvait donc laisser libre cours à la violence sans en pâtir. En ensanglantant le siècle précédent, les guerres de religion avaient retiré au christianisme sa légitimité à promouvoir la paix civile. Seul le roi en était désormais devenu le protecteur, en imposant la tolérance entre confessions antagonistes. La violence qu'il exerçait lui-même se voulait avant tout celle de la force du droit que lui procurait sa légitimité personnelle. Elle n'était plus religieuse, en dépit de son attachement propre au catholicisme et de l'existence d'un parti adverse composé de protestants. C'était celle de l'État.

Une autre gravure de Valdor, que nous fait découvrir Patrick Avrillas, témoigne pourtant de la difficulté à distinguer le droit et la religion dans les motivations des luttes. Elle illustre le " rétablissement des ecclésiastiques en Béarn ", en 1620. Il ne s'était agi alors que d'appliquer une clause de l'édit de Nantes de 1598 qu'on n'avait encore jamais osé imposer aux protestants de cette province. Pourtant Corneille commente ainsi la gravure : « Il tient dessoubz ses pieds l'hérésie estouffée. " De fait, la gravure représente le roi, bien raide, piétinant le cadavre de la " rébellion hérétique " auquel une chevelure longue et emmêlée donne l'apparence d'une hydre. Ce cadavre est-il vraiment plus rebelle qu'hérétique, puisqu'être hérétique n'était pas répréhensible en soi? La virulence de la représentation laisse pourtant place à une interprétation religieuse : quel vaincu méritait d'être ainsi littéralement aplati sous son vainqueur? Terrassé au terme d'un combat sans pitié? On dispose peut-être d'une confirmation de la lecture de cette image. Un siècle et demi plus tard en effet, bien après que l'édit de Nantes eut été révoqué, on érigea une sculpture semblable sur une nouvelle chaire de vérité installée en l'église Notre-Dame de Fontenay-le-Comte : même présentation en équerre, mais cette fois-ci de la Religion (catholique, apostolique et romaine) debout et terrassant l'Hérésie (protestante), qui gisait couchée sous ses pieds. L'image de Valdor avait ainsi eu valeur d'avertissement, tandis que, par la suite, la sculpture de Fontenay rendait compte du triomphe d'une confession sur l'autre. En attendant ce moment funeste, rien n'était joué, mais, depuis l'affaire de Béarn, en 1620, et surtout depuis le siège de La Rochelle puis la paix d'Alès en 1629 jusqu'à la révocation en 1685 , les protestants n'avaient plus disposé d'aucun moyen militaire. L'État moderne ne pouvait supporter cette forme d'autonomie - et de défiance - de ses sujets. La tolérance n'avait donc plus eu d'autre soutien que celui du roi, de sa parole et de sa bonne volonté. Louis XIV y mit fin. Louis XIII avait respecté la liberté de culte de ceux qu'il avait vaincus. 
Ce livre nous permet enfin d'approcher une réalité rarement mise en valeur, la grande jeunesse du roi. Certes, un roi de France est majeur à 13 ans, mais l'on sait qu'aucun n'a jamais exercé le pouvoir si tôt. Ainsi Louis XIII, né le 27 septembre 1601 , orphelin à 8 ans et demi, avait laissé gouverner sa mère. Le 21 novembre 1615 , il épousait Anne d'Autriche, de cinq jours plus âgée que lui. Ils avaient 14 ans tout juste, et ce mariage précoce troubla l'équilibre affectif du roi. Toutefois, dix-huit mois plus tard, le 24 avril 1617, à 15 ans et demi, il s'émancipait déjà de sa mère en ordonnant l'assassinat de son favori Concini et en la faisant enfermer dans un château dont elle mit deux ans à pouvoir s'évader. C'était encore un adolescent qui prenait le pouvoir, à une époque qui nous en donne certes d'autres exemples. Il l'exerça donc bien avant d'avoir rallié le précieux cardinal de Richelieu. Il avait cependant suffisamment joué à la guerre, rappelle Patrick Avrillas, pour connaître la cartographie, s'entendre à l'art des sièges et pour manifester, de 1620 à 1622 (de 19 à 21 ans), des qualités militaires nécessaires face à tous ceux qui ne croyaient pas à son autorité ou qui refusaient d'en reconnaître toute l'étendue. Il savait aussi manier la propagande et inciter les artistes à exalter sa gloire. De bibliothèque en musée, Patrick Avrillas a déniché un nombre incroyable de témoignages, jusqu'à cette médaille en faisant déjà un roi-soleil...

Thierry HECKMANN

FlAMEIN, Richard, La société fluide. Une histoire des mobilités sociales (XVII -XIXe siècles), Rennes, PUR, 2018, 354 p.

Dès la première ligne de la préface, Michel Biard annonce l'objectif de l'ouvrage de Richard Flamein : " cerner les contours de la bourgeoisie l'époque moderne ". Pour ce faire, l'auteur analyse minutieusement les mobilités sociales en prenant comme objet d'étude la dynastie Le Coulteux sur sept générations de 1600 à 1824 . Si l'histoire de la famille est bien connue grâce aux travaux de Michel Zylberberg, le présent ouvrage se concentre sur l'ascension de ses membres dans la bourgeoisie puis dans la noblesse tout en examinant minutieusement les éléments aboutissant à la création d'une identité propre.

Originaire de Rouen, la famille est également installée à Cadix mais c'est Paris qui prend de l'importance au XVIII ${ }^{\mathrm{e}}$ siècle avec le développement des activités bancaires. L'entreprise née en 1580 se spécialise tout d'abord dans le commerce des draps puis les investissements se diversifient. De nombreuses activités sont liées à la Bretagne (manufacture des tabacs de Morlaix, commerce de Saint-Malo, Compagnie des Indes). Avec l'enrichissement de la famille se pose le problème de la transmission du capital dynastique qui fait l'objet de la première partie de l'ouvrage (étude des liens familiaux, des choix résidentiels), alors que la seconde traite des facteurs de mobilité (réseaux d'affaires, sociabilité salonnière).

La transmission du capital vise naturellement à l'accumulation appuyée par des pratiques matrimoniales favorisant l'endogamie et le renforcement des réseaux. Les Le Coulteux mettent en place une organisation pour mobiliser le capi- 